\title{
COMPACT AND LOOSELY BOUND STRUCTURES IN LIGHT NUCLEI
}

\author{
O.L.Savchenko, A.I.Steshenko \\ Bogolyubov Institute for Theoretical Physics of the NAS of Ukraine \\ Metrolohichna Str., 14b, Kyiv-143, 03143 Ukraine
}

(November 5, 2018)

\begin{abstract}
A role of different components in the wave function of the weakly bound light nuclei states was studied within the framework of the cluster model, taking into account of orbitals "polarization". It was shown that a limited number of structures associated with the different modes of nucleon motion can be of great importance for such systems. Examples of simple and quite flexible trial wave functions are given for the nuclei ${ }^{8} \mathrm{Be},{ }^{6} \mathrm{He}$. Expressions for the microscopic wave functions of these nuclei were found and used for the calculation of basic nuclear characteristics, using well known central-exchange nucleon-nucleon potentials.
\end{abstract}

64.60.Cn, 81.30.Bx, 75.10.H

Typeset using REVTEX 


\section{INTRODUCTION.}

Theoretical consideration of strongly interacting many-particle systems, such as the atomic nuclei, has a number of difficulties, one of which is constructing of trial wave function. The wave function used should be characterized by proper set of the definite quantum numbers and corresponding symmetries, taking into account the most important physical modes of nucleon motion. It is usually assumed that structure of the constructed wave function is well known in the limitsd cases, i.e. in the case where a distance between fragments is very large or when we have a compact one-centre system. Hence, the main attention is paid to determination of correct interpolation expression for the wave function in the intermediate region.

Many authors have worked at simultaneous description of both "compact" and "friable" structures (see, for example, Ref. [1,2]). Several decades ago due to activity of Yu.A.Simonov, great attention was paid to the hyperspherical function [3] 5] method (the K-harmonic method) which was considered as a general solution method for the nuclear many-particle problem. But it was shown that its practical application is limited effectively to compact systems; moreover, the results obtained [6,7] hardly differ from the results calculated using the simplest functions of the oscillator shell model with one variation parameter.

Recently quite effective stochastic variational methods [8 12] for semirealistic nucleonnucleon (NN) potentials using a number of multicluster model basic functions have been developed for the case of the lightest $(A \leq 10)$ nuclei. They allow to find almost exact numerical solutions of many particle problems for some variants of NN-forces which are most frequently used in calculations of the light nuclei structure. Unfortunately, only numerical estimations of energies and radii are usually obtained due to complication of the calculations. Thus, results of such microscopic computations rarely can be used for estimation of other nuclear characteristics. Therefore, it is desirable to have many-particle wave function in evident form for real efficiency of the method. Moreover, if we have the wave function in the explicit form, we will be able to use it in calculations of different nuclear processes within the nuclear system.

To construct simple and quite flexible microscopic trial wave function of the nucleus which correctly describes both compact and weakly bound states, it is of prime importance at studing of exotic nuclei with anomalous ratio $\mathrm{N} / \mathrm{Z}$.

It is known that deformation effects are significant in nuclei with $A>4$. But concrete calculations are usually performed on the basis of spherical symmetry for cluster wave functions and detailed function for cluster-cluster motion. Recently, we have tried [13] to take into consideration the deformation effects in the framework of the many-particle oscillator shell model by using the variation principle (without taking into account the cluster modes). In the present paper we describe microscopic model which employs orbital "polarization" as well as cluster degrees of the nucleon motion and exact angular projection. The model can be used for description of both compact and weakly bound states in the light and medium light atomic nuclei.

Below we briefly outline the construction of the trial wave function (section II) and give all matrix elements (m.e.) of the basic physical operators needed for our computation (section [II]). The variational calculation results for the nuclei ${ }^{8} \mathrm{Be},{ }^{6} \mathrm{He}$ are given in section IV. 


\section{MANY-PARTICLE HAMILTONIAN AND TRIAL WAVE FUNCTIONS.}

Let us split considered A-nucleon system in two subsystems $\left(A=A_{1}+A_{2}\right)$ in such way that nucleon-nucleon interaction inside of each subsystem is a priori stronger then that between the two nucleons in the different fragments. We introduce the two fragments in order to simplify the account. In case if there are more fragments, the generalization can be written in a similar way. Note that nucleon motion mode defined by interaction between subsystems will be the "softest". Analysis of the experimental data for the considered nucleus allows to choose correct splitting of A-nucleon system into fragments. As a rule, the most interesting "physics" at low energies is caused by the soft mode which should be the most detailed in the framework of the chosen model. For example, description of the A-nucleus weak-bound state will be sufficient if we were able to obtain the basic nuclear characteristics as well as the decay $A=A_{1}+A_{2}$ threshold on the assumption that separated fragments $A_{1}$ and $A_{2}$ are known compact nuclei.

In general, every microscopic model is associated with its own effective NN-potential. The most known effective NN-potentials have been constructed in the framework of the models based on the shell model wave function with one variation parameter. It is clear that using such NN-potentials in the more advanced models can be uneffective. However, this usage can be quite correct if NN-potential was built on the basis of the compact nuclei properties (e.g., ones of the magic nuclei ${ }^{4} \mathrm{He},{ }^{16} \mathrm{O}$ or ${ }^{40} \mathrm{Ca}$ ). The results of calculations [13] for the magic nuclei ${ }^{4} \mathrm{He},{ }^{16} \mathrm{O}$ are rather similar for both simple and advanced model, therefore is valid to use mentioned above NN-potentials for the "polarized" orbitals model. Let us further consider the standard microscopic nuclear model [14 based on the many-nucleon Hamiltonian with two-particle central exchange interaction

$$
\hat{V}_{i j}(r)=\sum_{S, T=0,1} V_{2 S+1,2 T+1}(r) \cdot \hat{P}_{S T}(i j), \quad r \equiv\left|\vec{r}_{i}-\vec{r}_{j}\right|
$$

where the radius dependence of the potential components can be represented by Gaussian superposition, i.e.

$$
V_{2 S+1,2 T+1}(r)=\sum_{\nu=1}^{\nu_{p o t}} V_{2 S+1,2 T+1}^{[\nu]} \cdot \exp \left(-\frac{r^{2}}{\mu_{\nu}^{2}}\right)
$$

projection operator $\hat{P}$ in eq.(1) cut out the state of nucleon pair $(i j)$ with defined spin $S$ and isospin $T$.

When fragments are separated enough, the Hamiltonian $\hat{H}_{A}$ should turn into the sum of separated subsystem Hamiltonians plus Coulomb repulsion between them due to the short-ranged nature of the nuclear forces

$$
\hat{H}_{A} \Rightarrow \hat{H}_{A_{1}}+\hat{H}_{A_{2}}+\frac{Z_{1} Z_{2} e^{2}}{\left|\vec{R}_{1}-\vec{R}_{2}\right|}, \quad \vec{R}_{l}=\frac{1}{A_{l}} \sum_{i=1}^{A_{l}} \vec{r}_{i} ; \quad l=1,2
$$

i.e., the limiting cases are known.

The behaviour of the system in the intermediate range where altitude of vector $\vec{S}=\vec{R}_{1}-$ $\vec{R}_{2}$ significantly differs from zero, but is not large enough to neglect the nuclear interaction 
between particles in the different clusters, is open question. Hence, it is necessary to define dependency of the center-of-mass kinetic energy operator $\hat{T}_{c . m}$. on the stage of separation of the A-nucleus into fragments. We simulate $\hat{T}_{c . m}$. in this range by expression

$$
\begin{gathered}
\hat{T}_{c . m .}\left(\alpha_{S}\right)=-\frac{\hbar^{2}}{2 A m}\left[1+\alpha_{S}\left(\frac{A}{A_{1}}-1\right)\right] \cdot\left(\sum_{i=1}^{A_{1}} \vec{\nabla}_{i}\right)^{2}- \\
-\frac{\hbar^{2}}{2 A m}\left[1+\alpha_{S}\left(\frac{A}{A_{2}}-1\right)\right] \cdot\left(\sum_{j=A_{1}+1}^{A} \vec{\nabla}_{j}\right)^{2}-\frac{\hbar^{2}}{A m}\left(1-\alpha_{S}\right) \cdot \sum_{i=1}^{A_{1}} \sum_{j=A_{1}+1}^{A} \vec{\nabla}_{i} \vec{\nabla}_{j} .
\end{gathered}
$$

In our calculations the value of the parameter $\alpha_{S}\left(\left[0 \leq \alpha_{S} \leq 1\right]\right)$ in eq.(幽 was defined by the degree of importance of the Pauli principle in the A-nucleon system. Thus, $\alpha_{S}=0$ if antisymmetrization acts on the variables of all $\mathrm{A}$ nucleons and $\alpha_{S}=1$ in the other limiting case when the Pauli principle acts only on the nucleons inside the clusters.

A total trial wave function $\Psi_{A}$ is represented by superposition of a small number of determinant functions

$$
\Psi_{A}(1,2, \ldots, A)=\sum_{\nu} w_{\nu} \cdot \Psi_{\nu}
$$

where $\Psi_{\nu}$-components (normalized, but generally not orthogonal) are well known Slater determinants

$$
\Psi_{\nu}=\frac{f_{\nu}}{\sqrt{A !}} \operatorname{det}\left[\phi_{i}^{[\nu]}(j)\right], \quad f_{\nu}=\frac{1}{\sqrt{\operatorname{det}\left[<\phi_{i}^{[\nu]} \mid \phi_{j}^{[\nu]}>\right]}},
$$

filled by one-particle orbitals which have unificated form:

$$
\begin{gathered}
\phi_{i}(j)=\psi_{\vec{n}_{i}}\left(\vec{r}_{j} ; \vec{a}_{i}, \vec{R}_{i}\right) \cdot \chi_{\sigma \tau}(j) ; \quad \sum_{\sigma \tau} \int\left|\phi_{i}\right|^{2} d \vec{r}=1, \\
\psi_{\vec{n}}(\vec{r} ; \vec{a}, \vec{R})=\psi_{n^{x}}\left(x ; a, R^{x}\right) \psi_{n^{y}}\left(y ; b, R^{y}\right) \psi_{n^{z}}\left(z ; c, R^{z}\right), \\
\psi_{n}(x ; a, R)=\frac{1}{\sqrt{a \sqrt{\pi}} \cdot N(n ; R / a)} \cdot\left(\frac{x}{a}\right)^{n} \cdot \exp \left\{-\frac{(x-R)^{2}}{2 a^{2}}\right\}, \\
N(n ; R / a)=\sqrt{\left(\frac{R}{a}\right)^{2 n} \cdot F\left(-n,-n+\frac{1}{2} ; 0 ; \frac{a^{2}}{R^{2}}\right)},
\end{gathered}
$$

where $\chi_{\sigma \tau}$ is the spin-isospin function and $F(\alpha, \beta ; \gamma ; z)$ is the Gauss hyperspherical function [15].

Every component $\Psi_{\nu}$ in eq.(5) represents some characteristic structure or configuration of the considered system and is associated to some type of nuclear motion. We hope that the wave function $\Psi_{A}$ constructed in such way will be flexible enough at the reasonable number of the variation parameters $\left\{\vec{a}_{i}, \vec{R}_{i}\right\}$. 


\section{OVERLAP INTEGRALS.}

In order to perform the variational calculations it is necessary to obtain the matrix elements of some physical operators. Needed overlap integrals can be expressed in terms of "partial" m.e. on the basis of the function set (7) using known determinant function technique [16].

At the beginning, we consider overlap integral of the functions $\Psi_{\nu}$ with unity, i.e.

$$
<\Psi_{\nu} \mid \Psi_{\mu}>=f_{\nu} f_{\mu} \cdot \operatorname{det}\left[<\phi_{i}^{[\nu]} \mid \phi_{j}^{[\mu]}>\right] \equiv f_{\nu} f_{\mu} \cdot \operatorname{det}\left[\left(B_{[\nu, \mu]}\right)_{i j}\right]
$$

where the matrix $B_{[\nu, \mu]}$ is defined by

$$
\begin{gathered}
\left(B_{[\nu, \mu]}\right)_{i j}=<\chi_{\sigma_{i} \tau_{i}} \mid \chi_{\sigma_{j} \tau_{j}}>\int \psi_{\vec{n}_{i}}^{[\nu]}\left(\vec{r} ; \vec{a}_{i}, \vec{R}_{i}\right) \psi_{\vec{n}_{j}}^{[\mu]}\left(\vec{r} ; \vec{a}_{j}, \vec{R}_{j}\right) d \vec{r}= \\
=<\chi_{\sigma_{i} \tau_{i}} \mid \chi_{\sigma_{j} \tau_{j}}>\cdot g_{n_{i}^{x} n_{j}^{x}}\left(a_{i} R_{i}^{x} ; a_{j} R_{j}^{x}\right) g_{n_{i}^{y} n_{j}^{y}}\left(b_{i} R_{i}^{y} ; b_{j} R_{j}^{y}\right) g_{n_{i}^{z} n_{j}^{z}}\left(c_{i} R_{i}^{z} ; c_{j} R_{j}^{z}\right) .
\end{gathered}
$$

Evident expression of the partial m.e.

$$
g_{n_{1} n_{2}}\left(a_{1} R_{1} ; a_{2} R_{2}\right) \equiv \int_{-\infty}^{\infty} \psi_{n_{1}}\left(x ; a_{1}, R_{1}\right) \psi_{n_{2}}\left(x ; a_{2}, R_{2}\right) d x
$$

is given in the Appendix (see eq.(A1)).

The general expression [16] for one-particle operator matrix elements

$$
\frac{1}{<\Psi_{\nu} \mid \Psi_{\mu}>} \cdot<\Psi_{\nu}\left|\sum_{i=1}^{A} \hat{\Omega}_{i}\right| \Psi_{\mu}>=\sum_{i, j=1}^{A}<\phi_{i}^{[\nu]}\left|\hat{\Omega}_{1}\right| \phi_{j}^{[\mu]}>\cdot\left(B_{[\nu, \mu]}^{-1}\right)_{i j}
$$

allows to obtain:

1) m.e. for nuclear matter distribution $\rho(\vec{r})$

$$
\frac{1}{<\Psi_{\nu} \mid \Psi_{\mu}>} \cdot\left\langle\Psi_{\nu}\left|\sum_{i=1}^{A} \delta\left(\vec{r}-\vec{r}_{i}\right)\right| \Psi_{\mu}>=\sum_{i, j=1}^{A} \psi_{\vec{n}_{i}}^{[\nu]}\left(\vec{r} ; \vec{a}_{i}, \vec{R}_{i}\right) \psi_{\vec{n}_{j}}^{[\mu]}\left(\vec{r} ; \vec{a}_{j}, \vec{R}_{j}\right) \cdot\left(B_{[\nu, \mu]}^{-1}\right)_{i j} ;\right.
$$

2) m.e. for kinetic energy operator

$$
\frac{1}{<\Psi_{\nu} \mid \Psi_{\mu}>} \cdot\left\langle\Psi_{\nu}\left|-\sum_{i=1}^{A} \frac{\hbar^{2}}{2 m} \vec{\nabla}_{i}^{2}\right| \Psi_{\mu}>=\sum_{i, j=1}^{A}<\psi_{\vec{n}_{i}}^{[\nu]}\left|\hat{t}_{x}+\hat{t}_{y}+\hat{t}_{z}\right| \psi_{\vec{n}_{j}}^{[\mu]}>\cdot\left(B_{[\nu, \mu]}^{-1}\right)_{i j}\right.
$$

where

$$
\begin{gathered}
<\psi_{\vec{n}_{i}}\left|\hat{t}_{x}\right| \psi_{\vec{n}_{j}}>\equiv<\psi_{\vec{n}_{i}}^{[\nu]}\left(\vec{r} ; \vec{a}_{i}, \vec{R}_{i}\right)\left|-\frac{\hbar^{2}}{2 m} \frac{\partial^{2}}{\partial x^{2}}\right| \psi_{\vec{n}_{j}}^{[\mu]}\left(\vec{r} ; \vec{a}_{j}, \vec{R}_{j}\right)>= \\
=g_{n_{i}^{y} n_{j}^{y}}\left(b_{i} R_{i}^{y} ; b_{j} R_{j}^{y}\right) g_{n_{i}^{z} n_{j}^{z}}\left(c_{i} R_{i}^{z} ; c_{j} R_{j}^{z}\right) \cdot t_{n_{i}^{x} n_{j}^{x}}\left(a_{i} R_{i}^{x} ; a_{j} R_{j}^{x}\right) .
\end{gathered}
$$


The analogous expressions can be written for $\left\langle\psi_{\vec{n}_{i}}\left|\hat{t}_{y}\right| \psi_{\vec{n}_{j}}\right\rangle$ (substitution $x \rightarrow y ; a \rightarrow b$ ) and for $\left\langle\psi_{\vec{n}_{i}}\left|\hat{t}_{z}\right| \psi_{\vec{n}_{j}}>\right.$ (substitution $x \rightarrow z ; a \rightarrow c$ ). The matrix elements $t_{n^{\prime} n}\left(a^{\prime}, R^{\prime} ; a, R\right)$ employed in eq.(14) are given in the Appendix (see eq.(A2)). In eqs.(11)-(13) and below the inverse matrix

$$
B_{[\nu, \mu]}^{-1}=\left\|\left(B_{[\nu, \mu]}^{-1}\right)_{i j}\right\| .
$$

is used.

Further, let us use the general formula [16] for two-particle operators $\sum_{i, j=1}^{A} \hat{\Omega}_{i j}$

$$
\begin{gathered}
\frac{1}{<\Psi_{\nu} \mid \Psi_{\mu}>} \cdot<\Psi_{\nu}\left|\sum_{i, j=1}^{A} \hat{\Omega}_{i j}\right| \Psi_{\mu}>=\sum_{k^{\prime}, l^{\prime}, k, l=1}^{A}<\phi_{k^{\prime}}^{[\nu]} \phi_{l^{\prime}}^{[\nu]}\left|\hat{\Omega}_{12}\right| \phi_{k}^{[\mu]} \phi_{l}^{[\mu]}>\times \\
\times\left\{\left(B_{[\nu, \mu]}^{-1}\right)_{k k^{\prime}}\left(B_{[\nu, \mu]}^{-1}\right)_{l l^{\prime}}-\left(B_{[\nu, \mu]}^{-1}\right)_{k l^{\prime}}\left(B_{[\nu, \mu]}^{-1}\right)_{l k^{\prime}}\right\} .
\end{gathered}
$$

In the case of the central exchange NN-potential (11), (2) we can write for even-even nuclei after summing up on the spin-isospin variables

$$
\begin{gathered}
\frac{1}{<\Psi_{\nu} \mid \Psi_{\mu}>} \cdot<\Psi_{\nu}\left|\sum_{i, j=1}^{A} \hat{V}_{i j}\right| \Psi_{\mu}>= \\
=\sum_{i^{\prime}, i=1}^{Z} \sum_{j^{\prime}, j=1}^{Z} \sum_{l=1}^{l_{p o t}}<\psi_{\vec{n}_{i^{\prime}}}^{[\nu]}(1) \psi_{\vec{n}_{j^{\prime}}}^{[\nu]}(2)\left|\exp \left\{-\frac{\left|\vec{r}_{1}-\vec{r}_{2}\right|^{2}}{\mu_{l}^{2}}\right\}\right| \psi_{\vec{n}_{i}}^{[\mu]}(1) \psi_{\vec{n}_{j}}^{[\mu]}(2)>\times \\
\times\left\{X_{d}(l) \cdot\left(B_{[\nu, \mu]}^{-1}\right)_{i i^{\prime}}\left(B_{[\nu, \mu]}^{-1}\right)_{j j^{\prime}}-X_{e x}(l) \cdot\left(B_{[\nu, \mu]}^{-1}\right)_{i j^{\prime}}\left(B_{[\nu, \mu]}^{-1}\right)_{j i^{\prime}}\right\}+ \\
+\sum_{i^{\prime}, i=1}^{Z} \sum_{j^{\prime}, j=1}^{N} \sum_{l=1}^{l_{p o t}}<\psi_{\vec{n}_{i^{\prime}}}^{[\nu]}(1) \psi_{\vec{n}_{j^{\prime}}}^{[\nu]}(2)\left|\exp \left\{-\frac{\left|\overrightarrow{r_{1}}-\vec{r}_{2}\right|^{2}}{\mu_{l}^{2}}\right\}\right| \psi_{\vec{n}_{i}}^{[\mu]}(1) \psi_{\vec{n}_{j}}^{[\mu]}(2)>\times \\
\quad \times\left\{Y_{d}(l) \cdot\left(B_{[\nu, \mu]}^{-1}\right)_{i i^{\prime}}\left(B_{[\nu, \mu]}^{-1}\right)_{j j^{\prime}}-Y_{e x}(l) \cdot\left(B_{[\nu, \mu]}^{-1}\right)_{i j^{\prime}}\left(B_{[\nu, \mu]}^{-1}\right)_{j i^{\prime}}\right\}+ \\
+\sum_{i^{\prime}, i=1}^{N} \sum_{j^{\prime}, j=1}^{N} \sum_{l=1}^{l_{p o t}}<\psi_{\vec{n}_{i^{\prime}}}^{[\nu]}(1) \psi_{\vec{n}_{j^{\prime}}}^{[\nu]}(2)\left|\exp \left\{-\frac{\left|\vec{r}_{1}-\vec{r}_{2}\right|^{2}}{\mu_{l}^{2}}\right\}\right| \psi_{\vec{n}_{i}}^{[\mu]}(1) \psi_{\vec{n}_{j}}^{[\mu]]}(2)>\times \\
\quad \times\left\{X_{d}(l) \cdot\left(B_{[\nu, \mu]}^{-1}\right)_{i i^{\prime}}\left(B_{[\nu, \mu]}^{-1}\right)_{j j^{\prime}}-X_{e x}(l) \cdot\left(B_{[\nu, \mu]}^{-1}\right)_{i j^{\prime}}\left(B_{[\nu, \mu]}^{-1}\right)_{j i^{\prime}}\right\}
\end{gathered}
$$

where

$$
X_{d}(l)=\frac{3 V_{33}^{(l)}+V_{13}^{(l)}}{2}, \quad X_{e x}(l)=\frac{3 V_{33}^{(l)}-V_{13}^{(l)}}{2}
$$




$$
Y_{d}(l)=\frac{3 V_{33}^{(l)}+3 V_{31}^{(l)}+V_{13}^{(l)}+V_{11}^{(l)}}{2}, \quad Y_{e x}(l)=\frac{3 V_{33}^{(l)}-3 V_{31}^{(l)}-V_{13}^{(l)}+V_{11}^{(l)}}{2} .
$$

Due to the Gaussian dependence of the considered potential the partial matrix elements between the basis functions (7) are factorized on three terms, i.e.

$$
\begin{gathered}
<\psi_{\vec{n}_{i^{\prime}}}(1) \psi_{\vec{n}_{j^{\prime}}}(2)\left|\exp \left\{-\frac{\left|\vec{r}_{1}-\vec{r}_{2}\right|^{2}}{\mu^{2}}\right\}\right| \psi_{\vec{n}_{i}}(1) \psi_{\vec{n}_{j}}(2)>= \\
=j_{12}\left(n_{i^{\prime}}^{x} a_{i^{\prime}} R_{i^{\prime}}^{x}, n_{j^{\prime}}^{x} a_{j^{\prime}} R_{j^{\prime}}^{x}, \mu ; n_{i}^{x} a_{i} R_{i}^{x}, n_{j}^{x} a_{j} R_{j}^{x}\right) \times \\
\times j_{12}\left(n_{i^{\prime}}^{y} b_{i^{\prime}} R_{i^{\prime}}^{y}, n_{j^{\prime}}^{y} b_{j^{\prime}} R_{j^{\prime}}^{y} ; \mu ; n_{i}^{y} b_{i} R_{i}^{y}, n_{j}^{y} b_{j} R_{j}^{y}\right) j_{12}\left(n_{i^{\prime}}^{z} c_{i^{\prime}} R_{i^{\prime}}^{z}, n_{j^{\prime}}^{z} c_{j^{\prime}} R_{j^{\prime}}^{z} ; \mu ; n_{i}^{z} c_{i} R_{i}^{z}, n_{j}^{z} c_{j} R_{j}^{z}\right) .
\end{gathered}
$$

The evident expression for integral

$$
\begin{gathered}
j_{12}\left(n^{\prime} a_{1}^{\prime} R_{1}^{\prime}, m^{\prime} a_{2}^{\prime} R_{2}^{\prime} ; \mu ; n a_{1} R_{1}, m a_{2} R_{2}\right)= \\
=\int_{-\infty}^{\infty} \int_{-\infty}^{\infty} \psi_{n^{\prime}}\left(x_{1} ; a_{1}^{\prime} R_{1}^{\prime}\right) \psi_{m^{\prime}}\left(x_{2} ; a_{2}^{\prime} R_{2}^{\prime}\right) \exp \left\{-\frac{\left(x_{1}-x_{2}\right)^{2}}{\mu^{2}}\right\} \psi_{n}\left(x_{1} ; a_{1} R_{1}\right) \psi_{m}\left(x_{2} ; a_{2} R_{2}\right) d x_{1} d x_{2}
\end{gathered}
$$

is given in the Appendix (see eq. A44)).

In the context of described above calculations it is suitable to use the relation

$$
\frac{e^{2}}{r}=\frac{2 e^{2}}{\sqrt{\pi}} \int_{0}^{1} \exp \left(-\frac{r^{2}}{\mu_{o}^{2}}\right) \cdot \frac{d \tau}{\left(1-\tau^{2}\right)^{3 / 2}} ; \quad \mu_{o} \equiv \frac{1}{\tau} \sqrt{1-\tau^{2}} .
$$

for evaluation of the Coulomb repulsion between protons. Thus,

$$
\begin{aligned}
\frac{1}{<\Psi_{\nu} \mid \Psi_{\mu}>} & <\Psi_{\nu}\left|\sum_{i>j=1}^{Z} \frac{e^{2}}{\left|\vec{r}_{i}-\vec{r}_{j}\right|}\right| \Psi_{\mu}>=\frac{2 e^{2}}{\sqrt{\pi}} \sum_{i^{\prime}>j^{\prime}=1}^{Z} \sum_{i>j=1}^{Z} \int_{0}^{1} \frac{d \tau}{\left(1-\tau^{2}\right)^{3 / 2}} \times \\
\times & <\psi_{\vec{n}_{i^{\prime}}}(1) \psi_{\vec{n}_{j^{\prime}}}(2)\left|\exp \left\{-\frac{\left|\vec{r}_{1}-\vec{r}_{2}\right|^{2}}{\mu_{o}^{2}}\right\}\right| \psi_{\vec{n}_{i}}(1) \psi_{\vec{n}_{j}}(2)>\times \\
& \times\left\{2\left(B_{[\nu, \mu]}^{-1}\right)_{i i^{\prime}}\left(B_{[\nu, \mu]}^{-1}\right)_{j j^{\prime}}-\left(B_{[\nu, \mu]}^{-1}\right)_{i j^{\prime}}\left(B_{[\nu, \mu]}^{-1}\right)_{j i^{\prime}}\right\},
\end{aligned}
$$

i.e., in general, only integration over $\tau$ remains in the equation (21) for the Coulomb energy (all two-particle integrals for Gaussian potential are expressed in terms of regular functions).

The expression for center-of-mass kinetic energy has the form

$$
<\Psi_{\nu}\left|\hat{T}_{c . m .}\right| \Psi_{\mu}>=-\frac{\hbar^{2}}{2 A m}\left(<\Psi_{\nu}\left|\sum_{i=1}^{A} \vec{\nabla}_{i}^{2}\right| \Psi_{\mu}>+<\Psi_{\nu}\left|\sum_{i \neq j=1}^{A} \vec{\nabla}_{i} \vec{\nabla}_{j}\right| \Psi_{\mu}>\right)
$$


where the first term in the brackets is described by eqs.(13), (14); the other can be evaluated using general eq.(15) for two-particle operators, i.e.

$$
\begin{gathered}
\frac{1}{<\Psi_{\nu} \mid \Psi_{\mu}>} \cdot<\Psi_{\nu}\left|\sum_{i \neq j=1}^{A} \vec{\nabla}_{i} \vec{\nabla}_{j}\right| \Psi_{\mu}>= \\
=\sum_{i^{\prime} \neq j^{\prime}=1}^{A} \sum_{i \neq j=1}^{A}<\phi_{i^{\prime}}^{[\nu]}|\vec{\nabla}| \phi_{i}^{[\mu]}><\phi_{j^{\prime}}^{[\nu]}|\vec{\nabla}| \phi_{j}^{[\mu]}>\times \\
\times\left\{\left(B_{[\nu, \mu]}^{-1}\right)_{i i^{\prime}}\left(B_{[\nu, \mu]}^{-1}\right)_{j j^{\prime}}-\left(B_{[\nu, \mu]}^{-1}\right)_{i j^{\prime}}\left(B_{[\nu, \mu]}^{-1}\right)_{j i^{\prime}}\right\} ;
\end{gathered}
$$

the explicit expressions for m.e. $<\phi_{1}|\vec{\nabla}| \phi_{2}>$ are given in the Appendix (see eq.(A3)).

Next, we briefly consider projection of the determinant function on the state with defined angular momentum (the rotation effect in phenomenological models). It is obvious that considered above determinant functions $\Psi_{\nu}$ are superpositions of the states $\mid J M>$. In order to obtain the state with defined angular momentum $J$ and its projection $M$, we should define the projector $\hat{P}_{M K}^{J}$ to operate on the total wave function $\Psi_{A}$. It is suitable to write the projector $\hat{P}_{M K}^{J}$ in the Hill-Wheeler's integral form

$$
\hat{P}_{M K}^{J}=\frac{2 J+1}{8 \pi^{2}} \int d \Omega D_{M K}^{{ }^{*}}(\Omega) \cdot \hat{R}(\Omega)
$$

i.e. overlap integrals

$$
\frac{\int d \Omega D_{M M}^{J *}(\Omega)<\Psi_{A}|\hat{O}| \hat{R}(\Omega) \Psi_{A}>}{\int d \Omega D_{M M}^{J *}(\Omega)<\Psi_{A} \mid \hat{R}(\Omega) \Psi_{A}>}=\frac{\sum_{\nu \mu} w_{\nu} w_{\mu} \int d \Omega \cdot D_{M M}^{J{ }^{*}}(\Omega)<\Psi_{\nu}|\hat{O}| \hat{R}(\Omega) \Psi_{\mu}>}{\sum_{\nu \mu} w_{\nu} w_{\mu} \int d \Omega D_{M M}^{J}{ }^{*}(\Omega)<\Psi_{\nu} \mid \hat{R}(\Omega) \Psi_{\mu}>}
$$

should be evaluated to obtain eigenvalue of some physical operator $\hat{O}$. Transformation of the spatial and spin-isospin functions at rotation of the reference frames on Euler angles is performed by usual way [17]. A matrix $\tilde{B}$ can be defined analogously to eq.(9):

$$
\begin{gathered}
\left(\tilde{B}_{[\nu, \mu]}\right)_{i j} \equiv \int \psi_{\vec{n}_{i}}^{[\nu]}\left(\vec{r} ; \vec{a}_{i}, \vec{R}_{i}\right) \hat{R}(\Omega) \psi_{\vec{n}_{j}}^{[\mu]}\left(\vec{r} ; \vec{a}_{j}, \vec{R}_{j}\right) d \vec{r} \sim \\
\sim \iint d x d y d z\left(\frac{x}{a_{i}}\right)^{n_{i}^{x}}\left(\frac{y}{b_{i}}\right)^{n_{i}^{y}}\left(\frac{z}{c_{i}}\right)^{n_{i}^{z}}\left(\frac{\tilde{x}}{a_{j}}\right)^{n_{j}^{x}}\left(\frac{\tilde{y}}{b_{j}}\right)^{n_{j}^{y}}\left(\frac{\tilde{z}}{c_{j}}\right)^{n_{j}^{z}} \times \\
\times \exp \left\{-\frac{\left(x-R_{i}^{x}\right)^{2}}{2 a_{i}^{2}}-\frac{\left(y-R_{i}^{y}\right)^{2}}{2 b_{i}^{2}}-\frac{\left(z-R_{i}^{z}\right)^{2}}{2 c_{i}^{2}}-\frac{\left(\tilde{x}-R_{j}^{x}\right)^{2}}{2 a_{j}^{2}}-\frac{\left(\tilde{y}-R_{j}^{y}\right)^{2}}{2 b_{j}^{2}}-\frac{\left(\tilde{z}-R_{j}^{z}\right)^{2}}{2 c_{j}^{2}}\right\},
\end{gathered}
$$

$\tilde{x}=\tilde{x}_{1}, \tilde{y}=\tilde{x}_{2}, \tilde{z}=\tilde{x}_{3}$ are the Cartesian coordinates in the rotated frame of reference that can be written using the rotation [17] matrix $\left\|R_{m^{\prime} m}(\Omega)\right\|$, i.e.

$$
\tilde{x}_{m}=\sum_{m^{\prime}=1}^{3} R_{m^{\prime} m}(\Omega) \cdot x_{m^{\prime}}, \quad m=1,2,3 .
$$


Integration in eq.(27) can be performed completely by transforming quadratic form from the exponent to diagonal one.

Note that two-particle matrix elements containing four different orbitals can also be evaluated this way. Thus, in the case of m.e.

$$
<\psi_{\vec{n}_{i}}\left(\vec{r}_{1} ; \vec{a}_{i}, \vec{R}_{i}\right) \psi_{\vec{n}_{j}}\left(\vec{r}_{2} ; \vec{a}_{j}, \vec{R}_{j}\right)\left|\exp \left\{-\frac{\left(\vec{r}_{1}-\vec{r}_{2}\right)^{2}}{\mu^{2}}\right\} \hat{R}(\Omega)\right| \psi_{\vec{n}_{i^{\prime}}}\left(\vec{r}_{1} ; \vec{a}_{i^{\prime}}, \vec{R}_{i^{\prime}}\right) \psi_{\vec{n}_{j^{\prime}}}\left(\vec{r}_{2} ; \vec{a}_{j^{\prime}}, \vec{R}_{j^{\prime}}\right)>
$$

the quadratic form is constructed using 6-dimension vector $\vec{x}=\vec{x}\left(x_{1}, x_{2}, \ldots, x_{6}\right)$, where $\vec{r}_{1}=\vec{r}_{1}\left(x_{1}, x_{2}, x_{3}\right), \vec{r}_{2}=\vec{r}_{2}\left(x_{4}, x_{5}, x_{6}\right)$.

Hence, all integrations over particle coordinates can be performed completely. Unfortunately, resulting expressions in the case of the angular projection are too complicated to be written here. Integration over Euler angles $\Omega\left(\theta_{1}, \theta_{2}, \theta_{3}\right)$ have been carried out numerically, although it is possible that further investigations will allow to do it exactly.

\section{NUMERICAL RESULTS.}

There are few extremely significant systems in nuclear physics. One of them is nucleus ${ }^{8} \mathrm{Be}$ which have unique properties and is critical for the theory. Thus, ${ }^{8} \mathrm{Be}$ is 8 -nucleon weak-bound system whose ground state is very narrow resonance near the decay threshold. Therefore, calculation of the ${ }^{8}$ Be basic spectroscopic characteristics will be serious test for the considered microscopic model.

Due to the known symmetry of the nucleus ${ }^{8}$ Be we choose its wave function in form

$$
\Psi_{A}(1,2, \ldots, 8)=w_{1} \cdot \Psi_{1}+w_{2} \cdot\left(\Psi_{2}+\Psi_{3}\right)
$$

where $\Psi_{\nu}$ is Slater determinant (6) filled with simple orbitals:

a) $\nu=1$

$$
\Psi_{\vec{n}_{i}}^{[\nu=1]}(j)= \begin{cases}\psi_{0}\left(x_{j} ; \tilde{a}_{1}, 0\right) \psi_{0}\left(y_{j} ; \tilde{a}_{1}, 0\right) \psi_{0}\left(z_{j} ; \tilde{c}_{1}, 0\right), & i=1,2,3,4 \\ \psi_{0}\left(x_{j} ; \tilde{a}_{2}, 0\right) \psi_{0}\left(y_{j} ; \tilde{a}_{2}, 0\right) \psi_{1}\left(z_{j} ; \tilde{c}_{2}, 0\right), & i=5,6,7,8\end{cases}
$$

b) $\nu=2,3$

$$
\begin{gathered}
\Psi_{\vec{n}_{i}}^{[\nu=2]}(j)= \begin{cases}\psi_{0}\left(x_{j} ; a_{1}, 0\right) \psi_{0}\left(y_{j} ; a_{1}, 0\right) \psi_{0}\left(z_{j} ; c_{1},-s \cdot R\right), & i=1,2,3,4 ; \\
\psi_{0}\left(x_{j} ; a_{2}, 0\right) \psi_{0}\left(y_{j} ; a_{2}, 0\right) \psi_{1}\left(z_{j} ; c_{2}, R\right), & i=5,6,7,8 ;\end{cases} \\
\Psi_{\vec{n}_{i}}^{[\nu=3]}(j)= \begin{cases}\psi_{0}\left(x_{j} ; a_{1}, 0\right) \psi_{0}\left(y_{j} ; a_{1}, 0\right) \psi_{0}\left(z_{j} ; c_{1}, s \cdot R\right), & i=1,2,3,4 ; \\
\psi_{0}\left(x_{j} ; a_{2}, 0\right) \psi_{0}\left(y_{j} ; a_{2}, 0\right) \psi_{1}\left(z_{j} ; c_{2},-R\right), & i=5,6,7,8 ;\end{cases}
\end{gathered}
$$

The values $\left\{\tilde{a}_{1}, \tilde{c}_{1}, \tilde{a}_{2}, \tilde{c}_{2} ; a_{1}, c_{1}, a_{2}, c_{2}, R, s\right\}$ in these expressions are variation parameters defined by minimization of the total nuclear energy functional.

Let us consider our system at different values of the parameter $R$ in order to examine the system dependence on the distance between the fragments. The variation parameters were optimized for every value of $R$; when $R \geq R_{0}$ the orbitals in the determinant functions $\Psi_{\nu=2}$ 
and $\Psi_{\nu=3}$ are continuously transformed in order to obtain wave functions of two independent $\alpha$-particles at the limit $R \rightarrow \infty$, i.e.

$$
\begin{aligned}
& \Psi_{\vec{n}_{i}}^{[\nu=2]}(j)= \begin{cases}\psi_{0}\left(x_{j} ; a_{1}, 0\right) \psi_{0}\left(y_{j} ; a_{1}, 0\right) \psi_{0}\left(z_{j} ; c_{1},-s \cdot R\right), & i=1,2,3,4 ; \\
-\alpha(\xi) \cdot \psi_{0}\left(x_{j} ; a_{1}, 0\right) \psi_{0}\left(y_{j} ; a_{1}, 0\right) \psi_{0}\left(z_{j} ; c_{1}, s \cdot R\right)+ & i=5,6,7,8 ; \\
+\beta(\xi) \cdot \psi_{0}\left(x_{j} ; a_{2}, 0\right) \psi_{0}\left(y_{j} ; a_{2}, 0\right) \psi_{1}\left(z_{j} ; c_{2}, R\right), & i=1,2,3,4 ;\end{cases} \\
& \Psi_{\vec{n}_{i}{ }^{[\nu=3]}}(j)= \begin{cases}\psi_{0}\left(x_{j} ; a_{1}, 0\right) \psi_{0}\left(y_{j} ; a_{1}, 0\right) \psi_{0}\left(z_{j} ; c_{1}, s \cdot R\right), & \\
\alpha(\xi) \cdot \psi_{0}\left(x_{j} ; a_{1}, 0\right) \psi_{0}\left(y_{j} ; a_{1}, 0\right) \psi_{0}\left(z_{j} ; c_{1},-s \cdot R\right)+ & \\
+\beta(\xi) \cdot \psi_{0}\left(x_{j} ; a_{2}, 0\right) \psi_{0}\left(y_{j} ; a_{2}, 0\right) \psi_{1}\left(z_{j} ; c_{2},-R\right), & i=5,6,7,8 ;\end{cases}
\end{aligned}
$$

The functions $\alpha(\xi), \beta(\xi)$ are defined by

$$
\alpha(\xi)=\frac{\xi}{\sqrt{1+2 \xi \sqrt{1-\xi^{2}}}}, \quad \beta(\xi)=\frac{\sqrt{1-\xi^{2}}}{\sqrt{1+2 \xi \sqrt{1-\xi^{2}}}}, \quad \xi \equiv \frac{R-R_{0}}{\eta_{0} \cdot R_{0}}, \quad 0 \leq \xi \leq 1 .
$$

Choice of $R_{0}$ and $\eta_{0}$ in eq.(36) accords to choice of interval $\left[R_{0},\left(\eta_{0}+1\right) R_{0}\right]$ where one of the $\alpha$-clusters "loses" its excitation. Next we define the antisymmetrization of the wave function, if $R \leq R_{0}$ then the Pauli principle acts on all $\mathrm{A}$ nucleons in the nucleus. In the range $\left[R_{0},\left(\eta_{0}+1\right) R_{0}\right]$ the antisymmetrization between particles in the different clusters is gradually "switched off". Moreover, both "losing" of the excitation and "switching off" of the Pauli principle action are simulated by the same functions $\alpha(\xi), \beta(\xi)$.

The total wave function of the system in the considered range $\left[R_{0},\left(\eta_{0}+1\right) R_{0}\right]$ is presented by

$$
\Psi_{A}(1,2, \ldots, 8) \sim \beta(\xi) \cdot w_{1}^{0} \Psi_{1}+[1+\alpha(\xi)] \cdot w_{2}^{0} \cdot\left(\Psi_{2}+\Psi_{2}\right),
$$

where $w_{1}^{0}, w_{2}^{0}$ are weights of the according determinant functions at $R=R_{0}$. The parameter $\alpha_{S}$ in the microscopic Hamiltonian (see eq.(四)) can be defined by

$$
\alpha_{S}=\alpha_{S}(R)= \begin{cases}0, & R<R_{0} \\ \alpha(\xi), & R_{0} \leq R \leq\left(\eta_{0}+1\right) R_{0} \\ 1, & R>\left(\eta_{0}+1\right) R_{0}\end{cases}
$$

In order to evaluate the overlap integrals corresponding to some physical quantity it is necessary to calculate the matrix $B_{[\nu, \mu]}$ and the inverse matrix $B_{[\nu, \mu]}^{-1}$. In the case of nucleus ${ }^{8} \mathrm{Be}$ these matrices are in general

$$
B_{[\nu, \mu]}=\left|\begin{array}{cccccccc}
\alpha_{\nu \mu} & 0 & 0 & 0 & \gamma_{\nu \mu} & 0 & 0 & 0 \\
0 & \alpha_{\nu \mu} & 0 & 0 & 0 & \gamma_{\nu \mu} & 0 & 0 \\
0 & 0 & \alpha_{\nu \mu} & 0 & 0 & 0 & \gamma_{\nu \mu} & 0 \\
0 & 0 & 0 & \alpha_{\nu \mu} & 0 & 0 & 0 & \gamma_{\nu \mu} \\
\delta_{\nu \mu} & 0 & 0 & 0 & \beta_{\nu \mu} & 0 & 0 & 0 \\
0 & \delta_{\nu \mu} & 0 & 0 & 0 & \beta_{\nu \mu} & 0 & 0 \\
0 & 0 & \delta_{\nu \mu} & 0 & 0 & 0 & \beta_{\nu \mu} & 0 \\
0 & 0 & 0 & \delta_{\nu \mu} & 0 & 0 & 0 & \beta_{\nu \mu}
\end{array}\right| ; \quad \operatorname{det}\left\|\left(B_{[\nu, \mu]}\right)_{i j}\right\|=\zeta_{0}^{4}
$$




$$
B_{[\nu, \mu]}^{-1}=\left|\begin{array}{cccccccc}
\beta_{\nu \mu} / \zeta_{0} & 0 & 0 & 0 & -\gamma_{\nu \mu} / \zeta_{0} & 0 & 0 & 0 \\
0 & \beta_{\nu \mu} / \zeta_{0} & 0 & 0 & 0 & -\gamma_{\nu \mu} / \zeta_{0} & 0 & 0 \\
0 & 0 & \beta_{\nu \mu} / \zeta_{0} & 0 & 0 & 0 & -\gamma_{\nu \mu} / \zeta_{0} & 0 \\
0 & 0 & 0 & \beta_{\nu \mu} / \zeta_{0} & 0 & 0 & 0 & -\gamma_{\nu \mu} / \zeta_{0} \\
-\delta_{\nu \mu} / \zeta_{0} & 0 & 0 & 0 & \alpha_{\nu \mu} / \zeta_{0} & 0 & 0 & 0 \\
0 & -\delta_{\nu \mu} / \zeta_{0} & 0 & 0 & 0 & \alpha_{\nu \mu} / \zeta_{0} & 0 & 0 \\
0 & 0 & -\delta_{\nu \mu} / \zeta_{0} & 0 & 0 & 0 & \alpha_{\nu \mu} / \zeta_{0} & 0 \\
0 & 0 & 0 & -\delta_{\nu \mu} / \zeta_{0} & 0 & 0 & 0 & \alpha_{\nu \mu} / \zeta_{0}
\end{array}\right| .
$$

Evident expressions for $\alpha_{\nu \mu}, \beta_{\nu \mu}, \gamma_{\nu \mu}, \delta_{\nu \mu}$ can be obtained by using the definitions (30)-(34) (see eqs.(A6) in the Appendix).

Concrete calculations on the basis of the above considered equations have been performed for the case of some well known variants of the central exchange NN-potentials. Thus, the results for Volkov's NN-potential [18] (the 1st variant case) are accurate. Some of the results

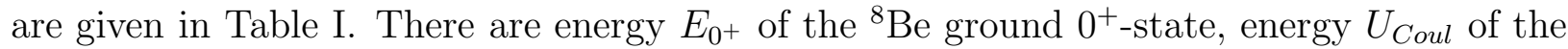
Coulomb repulsion between protons, r.m.s. matter radii $\left\langle r^{2}\right\rangle^{1 / 2}$ as well as optimized values of the variation parameters $\left\{a_{i}, b_{i}, c_{i}, \tilde{a}_{i}, \tilde{b}_{i}, \tilde{c}_{i}, R, s\right\}$ in the determinant functions $\Psi_{\nu}$ and its non-normalized weights $\left\{w_{i}\right\}$. In the case of N1 the wave function (30) contains 10 nonlinear variation parameters and describes both orbitals "polarization" and cluster structure of the nucleus ${ }^{8} \mathrm{Be}$. In the case of $\mathrm{N} 2$, the superposition of two determinant functions

$$
\begin{gathered}
\Psi_{A}=(1,2, \ldots, A)=w_{1} \cdot \Psi_{1}\left(\left\{\vec{r}_{i}\right\} ; \tilde{a}_{1}=\tilde{b}_{1}, \tilde{c}_{1} ; \tilde{a}_{2}=\tilde{b}_{2}, \tilde{c}_{2}\right)+ \\
+w_{4} \cdot \Psi_{4}\left(\left\{\vec{r}_{i}\right\} ; \tilde{\tilde{a}}_{1}=\tilde{\tilde{b}}_{1}, \tilde{\tilde{c}}_{1} ; \tilde{\tilde{a}}_{2}=\tilde{\tilde{b}}_{2}, \tilde{\tilde{c}}_{2}\right) .
\end{gathered}
$$

taking into account only orbitals "polarization" has been used. Column N3 corresponds to the previous case with neglecting of the deformation, i.e.

$$
\Psi_{A}=(1,2, \ldots, A)=w_{1} \cdot \Psi_{1}\left(\left\{\vec{r}_{i}\right\} ; \tilde{a}\right)+w_{4} \cdot \Psi_{4}\left(\left\{\vec{r}_{i}\right\} ; \tilde{\tilde{a}}\right)
$$

The values of column N4 are calculated on the basis of one determinant function with different degree of "polarization" for s- and p-orbitals (4 variation parameters: $\tilde{a}_{1}=\tilde{b}_{1}$, $\left.\tilde{c}_{1}, \tilde{a}_{2}=\tilde{b}_{2}, \tilde{c}_{2}\right)$. In the case of N5 we have used one determinant function with the same deformation for s- and p-orbitals (2 parameters: $\tilde{a}=\tilde{b}, \tilde{c})$. The simplest approach is microscopic $\mathrm{SU}(3)$-model with one variation parameter $\tilde{a}=\tilde{b}=\tilde{c}$ that corresponds to column N6. The data in column N7 have been calculated using the most complicated wave function $\Psi_{A}$. It differs from one of the case N1 by additional shell-type determinant function $\Psi_{\nu=4}$ filled by one-particle states containing independent variation parameters $\tilde{\tilde{a}}_{1}=\tilde{\tilde{b}}_{1}, \tilde{\tilde{c}}_{1}$, $\tilde{\tilde{a}}_{2}=\tilde{\tilde{b}}_{2}, \tilde{\tilde{c}}_{2}$. This approach employs 14 nonlinear variation parameters. And, finally, data in the column N8 have been evaluated using the previous function $\Psi_{A}$, but without angular projection.

First we consider numerical results obtained with one-center functions $\Psi_{A}$ (models $\mathrm{N} 2-$ N6). The data in Table 1 show that the most binding energy growth is caused by taking into account the oscillator field deformation (compare models N6 and N5). Independent orbital 
"polarization" is also significant (compare models N5 and N4). If the nonlinear variation parameters $\left\{a_{i}, b_{i}, c_{i}\right\}$ are the same (i.e. deformation is neglected) than expanding of the basis from 1 to 2 determinant functions $\Psi_{\nu}$ does not practically change the nuclear energy $\mathrm{E}$ (comp. models N3 and N6). Moreover, our estimations show that the employment of two, three and more determinant functions $\Psi_{\nu}$ with the same $\mathrm{SU}(3)$-symmetry does not allow to reach significant advance even if all considered s- and p-orbital deformations are taken into account (comp. models N2 and N4). Hence, the deformation effects can be considered using only one one-center Slater determinant. More effective is expansion of the variation basis by cluster wave function. It enhances the ${ }^{8} \mathrm{Be}$ nuclear energy by approximately $3 \mathrm{MeV}$ (comp. models N1, N4, N2).

Fig. 11 shows ${ }^{8}$ Be mass distribution $\rho(\vec{r})$ evaluated by using the wave function (30). The optimum variation parameters correspond to model N1 (see Table I). The calculations are carried out in the nuclear center-of-mass reference frames. Surface $(x, y=0, z)$ is displayed in Fig. 1 due to axial symmetry of the distribution $\rho(\vec{r})$. We can see from Fig. 1 that distance between density maximums (centers of mass for two $\alpha$-particles) is $D \approx 4.2 \mathrm{fm}$. Density of junction between $\alpha$-particles is approximately $0.7 \rho_{\max }$. The distribution $\rho(\vec{r})$ obtained in the framework of the other considered models is rather similar to Fig. 1, although distance $D$ and width of junction can be essentially different.

Evaluated parameter $R$ dependence of the ${ }^{8}$ Be total energy functional $f_{E}\left(R,\left\{a_{i}, b_{i}, c_{i}\right\}\right)$ is plotted in Fig. 2. The shown dependence have been calculated under condition that for every value $R$ functional $f_{E}$ is optimized to the other variation parameters. Such computations can be useful for estimations of the nucleus-nucleus potentials. Note that in a range where clusters overlap each other, the distance $D$ between density maximums can essentially differ from its asymptotic value which is equal to $D_{a s}=R(1+s)$ in our case. Fig. 3 shows dependence $D=f(R)$ obtained for model N1. We can see that the distance $D$ can slightly decrease at $0 \leq R \leq 1.5 \mathrm{fm}$ and only at $R>2 \mathrm{fm}$ the $R$ dependence of $D$ is almost linear.

Table $\mathbb{~ I 1 ] ~ c o n t a i n s ~ c h a r a c t e r i s t i c s ~ o f ~ t h e ~}{ }^{6} \mathrm{He}$ ground $0^{+}$-state calculated using three variants of NN-potentials: V1 from Ref. [18], BB1 from Ref. [19] and G5 from Ref. [20]. Computations are carried out in the framework of two models. Wave function of model $\mathrm{I}$ is a Slater determinant for 6 nucleons filled by orbitals (31), and contains four variation parameters: $\tilde{a}_{1}=\tilde{b}_{1}, \tilde{c}_{1}$ for first four orbitals and $\tilde{a}_{2}=\tilde{b}_{2}, \tilde{c}_{2}$ for the last two orbitals. Wave function of the second model is superposition of a few Slater determinants, similarly to the ${ }^{8} \mathrm{Be}$ : one-center determinant (model I) and two-center determinants with centers in points $\vec{R}_{1}=(0,0, \pm R)$ and $\vec{R}_{2}=(0,0, \mp s R)$. This model contains 10 nonlinear variation parameters whose optimum values for the considered variants of $\mathrm{NN}$-forces are given in Table III.

It should be remembered, that a core in ${ }^{6} \mathrm{He}$ is nucleus ${ }^{4} \mathrm{He}$. Its energy calculated using simple $\mathrm{SU}(3)$-model function is equal for three variants of NN-forces: $E_{\alpha}(\mathrm{V} 1)=-27.09$ $\mathrm{MeV}, E_{\alpha}(\mathrm{BB} 1)=-27.37 \mathrm{MeV}, E_{\alpha}(\mathrm{G} 5)=-28.29 \mathrm{MeV}$. Comparison of these values with ${ }^{6} \mathrm{He}$ ground state energies in Table $\mathbb{I I}$ shows that two neutrons are bound only in the case of model II and NN-potentials V1 and G5. Therefore, we can conclude that wave function of model II describes the real systems more accurate than one-center function of model I.

Note that authors don't claim superexact description of the considered here nuclear systems which has been already studied profoundly by many authors (see, for example, Refs. [9, 10]). The purpose of this study is to clarify the question about significance of 
"compact" and "loosely bound" structures at consideration of nuclear states using simple trial wave functions.

Performed analysis allows to draw up some general scheme for the construction of the simplest and quite flexible trial wave function of weakly bound state as well as a strategy

for concrete computation. First, possible significance of the deformation in formation of considered nucleus state is roughly estimated in the framework of the one-center determinant function model. Next, cluster degrees of freedom are taken into account. Hierarchy of the nucleon motion modes can be determined using non-projected wave functions $\Psi_{A}$ that significantly reduces computations. Moreover, such reduced evaluations give approximate optimum values for almost all variation parameters (for example, compare corresponding parameters of models N7 and N8 in Table (1). It is obvious that this fact is especially significant in order to find the global minimum of the total nuclear energy functional by employing projected functions $\Psi_{A}^{J M}(1,2, \ldots, A)$.

\section{APPENDIX:}

The evident expression (7) allows to calculate:

1) partial m.e. (10)

$$
\begin{gathered}
g_{n_{1} n_{2}}\left(a_{1}, R_{1} ; a_{2}, R_{2}\right)=\frac{g_{0}}{N\left(n_{1} ; \frac{R_{1}}{a_{1}}\right) N\left(n_{2} ; \frac{R_{2}}{a_{2}}\right)} \cdot\left(\frac{Z_{0}}{X_{0} Y_{0}}\right)^{n_{1}+n_{2}} \times \\
\times F\left(-\frac{n_{1}+n_{2}}{2},-\frac{n_{1}+n_{2}}{2}+\frac{1}{2} ; 0 ; \frac{1}{Z_{0}^{2}}\right), \\
g_{0} \equiv \sqrt{X_{0} Y_{0}} \cdot \exp \left\{-\frac{\left(R_{1}-R_{2}\right)^{2}}{2\left(a_{1}^{2}+a_{2}^{2}\right)}\right\} ; \quad X_{0}=\sqrt{\frac{2 a_{2}^{2}}{a_{1}^{2}+a_{2}^{2}} ; \quad Y_{0}=\sqrt{\frac{2 a_{1}^{2}}{a_{1}^{2}+a_{2}^{2}}} ;} \\
Z_{0}=\frac{1}{2}\left(\frac{R_{1}}{a_{1}} X_{0}+\frac{R_{2}}{a_{2}} Y_{0}\right) ;
\end{gathered}
$$

2) m.e. (14) used for evaluation of the kinetic energy

$$
\begin{gathered}
t_{n^{\prime} n}\left(a^{\prime}, R^{\prime} ; a, R\right) \equiv<\psi_{n^{\prime}}\left(x ; a^{\prime}, R^{\prime}\right)\left|-\frac{\hbar^{2}}{2 m} \frac{\partial^{2}}{\partial x^{2}}\right| \psi_{n}(x ; a, R)>= \\
=-\frac{\hbar^{2}}{2 m a^{2}} \cdot \frac{1}{N\left(n ; \frac{R}{a}\right)}\left\{n(n-1) N\left(n-2, \frac{R}{a}\right) g_{n^{\prime}, n-2}\left(a^{\prime}, R^{\prime} ; a, R\right)+\right. \\
+2 n \frac{R}{a} N\left(n-1, \frac{R}{a}\right) g_{n^{\prime}, n-1}\left(a^{\prime}, R^{\prime} ; a, R\right)+\left(\frac{R^{2}}{a^{2}}-2 n-1\right) N\left(n, \frac{R}{a}\right) g_{n^{\prime}, n}\left(a^{\prime}, R^{\prime} ; a, R\right)- \\
\left.-2 \frac{R}{a} N\left(n+1, \frac{R}{a}\right) g_{n^{\prime}, n+1}\left(a^{\prime}, R^{\prime} ; a, R\right)+N\left(n+2, \frac{R}{a}\right) g_{n^{\prime}, n+2}\left(a^{\prime}, R^{\prime} ; a, R\right)\right\} ;
\end{gathered}
$$




$$
\begin{gathered}
<\phi_{1}|\vec{\nabla}| \phi_{2}>=<\phi_{1}\left|\frac{\partial}{\partial x} \phi_{2}>\cdot \vec{e}_{x}+<\phi_{1}\right| \frac{\partial}{\partial y} \phi_{2}>\cdot \vec{e}_{y}+<\phi_{1} \mid \frac{\partial}{\partial z} \phi_{2}>\cdot \vec{e}_{z}, \\
<\phi_{1}\left|\frac{\partial}{\partial x} \phi_{2}>\equiv<\psi_{\vec{n}}(\vec{r} ; \overrightarrow{\tilde{a}}, \overrightarrow{\widetilde{R}})\right| \frac{\partial}{\partial x} \psi_{\vec{n}}(\vec{r} ; \vec{a}, \vec{R})>=g_{\tilde{n}_{y} n_{y}}\left(\tilde{b}, \tilde{R}^{y} ; b R^{y}\right) g_{\tilde{n}_{z} n_{z}}\left(\tilde{c}, \tilde{R}^{z} ; c R^{z}\right) \times \\
\times\left[n^{x} \frac{N\left(n^{x} ; R^{x} / a\right)}{N\left(n^{x}-1 ; R^{x} / a\right)} g_{\tilde{n}^{x}, n^{x}-1}\left(\tilde{a}, \tilde{R}^{x} ; a R^{x}\right)+\frac{R^{x}}{a} g_{\tilde{n}^{x}, n^{x}}\left(\tilde{a}, \tilde{R}^{x} ; a R^{x}\right)-\right. \\
\left.-\frac{N\left(n^{x} ; R^{x} / a\right)}{N\left(n^{x}+1 ; R^{x} / a\right)} g_{\tilde{n}^{x}, n^{x}+1}\left(\tilde{a}, \tilde{R}^{x} ; a R^{x}\right)\right],
\end{gathered}
$$

equations for $\left\langle\phi_{1}\right| \frac{\partial}{\partial y} \phi_{2}>$ and $<\phi_{1} \mid \frac{\partial}{\partial z} \phi_{2}>$ can be obtained from (A3) after obvious substitution $a \rightarrow b, R^{x} \rightarrow R^{y}$ and $a \rightarrow c, R^{x} \rightarrow R^{z}$;

3) 2-dimension integral (19) determining the potential energy of NN-interaction

$$
\begin{gathered}
j_{12}\left(\tilde{n} \tilde{a}_{1} \tilde{R}_{1}, \tilde{m} \tilde{a}_{2} \tilde{R}_{2} ; \mu ; n a_{1} R_{1}, m a_{2} R_{2}\right)=\frac{1}{\sqrt{\tilde{a}_{1} \tilde{a}_{2} a_{1} a_{2}}} \cdot \frac{\mu^{2}}{\eta_{0}} \times \\
\times\left(\frac{1}{2 \tilde{a}_{1}}\right)^{\tilde{n}} N\left(\tilde{n} ; \frac{\tilde{R}_{1}}{\tilde{a}_{1}}\right)\left(\frac{1}{2 \tilde{a}_{2}}\right)^{\tilde{m}} N\left(\tilde{m} ; \frac{\tilde{R}_{2}}{\tilde{a}_{2}}\right)\left(\frac{1}{2 a_{1}}\right)^{n} N\left(n ; \frac{R_{1}}{a_{1}}\right)\left(\frac{1}{2 a_{2}}\right)^{m} N\left(m ; \frac{R_{2}}{a_{2}}\right) \times \\
\times\left(\frac{\mu}{\xi_{0} \eta_{0}}\right)^{\tilde{n}+n}\left(\eta \frac{\xi_{0}}{\eta_{0}}\right)^{\tilde{m}+m} \cdot \exp \{+a r g\} \sum_{l-0}^{\tilde{n}+n} \frac{\eta_{0}^{l}(\tilde{n}+n) !}{l !(\tilde{n}+n-l) !} \sum_{k=0}^{\left[\frac{l}{2}\right]} \frac{l !}{k !(l-2 k) !} \times \\
\times\left[\frac{\mu}{\xi_{0}}\left(\frac{R_{1}}{a_{1}^{2}}+\frac{\tilde{R}_{1}}{\tilde{a}_{1}^{2}}\right)\right]^{l-2 k}\left[\frac{\tilde{n}+n+\tilde{m}+m-l}{2}\right] \\
\sum_{i=0}^{2} \frac{(\tilde{n}+n+\tilde{m}+m-l) !}{i !(\tilde{n}+n+\tilde{m}+m-l-2 i) !} \times \\
\times\left[\frac{\mu}{\xi_{0} \eta_{0}}\left(\frac{R_{1}}{a_{1}^{2}}+\frac{\tilde{R}_{1}}{\tilde{a}_{1}^{2}}\right)+\frac{\mu \xi_{0}}{\eta_{0}}\left(\frac{R_{2}}{a_{2}^{2}}+\frac{\tilde{R}_{2}}{\tilde{a}_{2}^{2}}\right)\right]^{\tilde{n}+n+\tilde{m}+m-l-2 i}
\end{gathered}
$$

where

$$
\begin{gathered}
\xi_{0}=\left[1+\frac{\mu^{2}}{2 a_{1}^{2}}+\frac{\mu^{2}}{2 a_{2}^{2}}\right]^{\frac{1}{2}}, \\
\eta_{0}=\left[\frac{\mu^{2}}{2 a_{1}^{2}}+\frac{\mu^{2}}{2 \tilde{a}_{1}^{2}}+\frac{\mu^{2}}{2 a_{2}^{2}}+\frac{\mu^{2}}{2 \tilde{a}_{2}^{2}}+\left(\frac{\mu^{2}}{2 a_{1}^{2}}+\frac{\mu^{2}}{2 \tilde{a}_{1}^{2}}\right)\left(\frac{\mu^{2}}{2 a_{2}^{2}}+\frac{\mu^{2}}{2 \tilde{a}_{2}^{2}}\right)\right]^{\frac{1}{2}}, \\
\arg =-\frac{1}{2}\left(\frac{\tilde{R}_{1}^{2}}{\tilde{a}_{1}^{2}}+\frac{R_{1}^{2}}{a_{1}^{2}}+\frac{\tilde{R}_{2}^{2}}{\tilde{a}_{2}^{2}}+\frac{R_{2}^{2}}{a_{2}^{2}}\right)+
\end{gathered}
$$




$$
\begin{gathered}
+\frac{1}{4} \frac{\left(1+\frac{\mu^{2}}{2 \tilde{a}_{2}^{2}}+\frac{\mu^{2}}{2 a_{2}^{2}}\right)\left(\frac{\mu \tilde{R}_{1}}{\tilde{a}_{1}^{2}}+\frac{\mu R_{1}}{a_{1}^{2}}\right)^{2}+\left(1+\frac{\mu^{2}}{2 \tilde{a}_{1}^{2}}+\frac{\mu^{2}}{2 a_{1}^{2}}\right)\left(\frac{\mu \tilde{R}_{2}}{\tilde{a}_{2}^{2}}+\frac{\mu R_{2}}{a_{2}^{2}}\right)^{2}}{\left(1+\frac{\mu^{2}}{2 \tilde{a}_{1}^{2}}+\frac{\mu^{2}}{2 a_{1}^{2}}\right)\left(1+\frac{\mu^{2}}{2 \tilde{a}_{2}^{2}}+\frac{\mu^{2}}{2 a_{2}^{2}}\right)-1}+ \\
+\frac{1}{2} \frac{\left(\frac{\mu \tilde{R}_{1}}{\tilde{a}_{1}^{2}}+\frac{\mu R_{1}}{a_{1}^{2}}\right)\left(\frac{\mu \tilde{R}_{2}}{\tilde{a}_{2}^{2}}+\frac{\mu R_{2}}{a_{2}^{2}}\right)}{\left(1+\frac{\mu^{2}}{2 \tilde{a}_{1}^{2}}+\frac{\mu^{2}}{2 a_{1}^{2}}\right)\left(1+\frac{\mu^{2}}{2 \tilde{a}_{2}^{2}}+\frac{\mu^{2}}{2 a_{2}^{2}}\right)-1} .
\end{gathered}
$$

Due to definitions (28)-(32) the $B_{[\nu, \mu]}$ matrix elements (see eq.(36) ) can be expressed in terms of functions $g_{n m}$ from eq.(A1):

$$
\begin{aligned}
& \alpha_{11}=\alpha_{22}=1, \quad \alpha_{12}=\alpha_{21}=g_{00}^{2}\left(\tilde{a}_{1}, 0 ; \tilde{a}_{11}, 0\right) g_{00}\left(\tilde{c}_{1}, 0 ; \tilde{c}_{11}, 0\right), \\
& \beta_{11}=\beta_{22}=1, \quad \beta_{12}=\beta_{21}=g_{00}^{2}\left(\tilde{a}_{2}, 0 ; \tilde{a}_{22}, 0\right) g_{11}\left(\tilde{c}_{2}, 0 ; \tilde{c}_{22}, 0\right), \\
& \gamma_{11}=\gamma_{22}=0, \quad \gamma_{12}=\gamma_{21}=g_{00}^{2}\left(\tilde{a}_{1}, 0 ; \tilde{a}_{22}, 0\right) g_{01}\left(\tilde{c}_{1}, 0 ; \tilde{c}_{22}, 0\right), \\
& \delta_{11}=\delta_{22}=0, \quad \delta_{12}=\delta_{21}=g_{00}^{2}\left(\tilde{a}_{2}, 0 ; \tilde{a}_{11}, 0\right) g_{10}\left(\tilde{c}_{2}, 0 ; \tilde{c}_{11}, 0\right) .
\end{aligned}
$$




\section{REFERENCES}

[1] A.I.Baz and M.V.Zhukov, Yad.Fiz. 16, 60 (1972).

[2] A.I.Baz and M.V.Zhukov, Yad.Fiz. 16, 958 (1972).

[3] Yu.A.Simonov, Yad.Fiz. 3, 630 (1966).

[4] A.M.Badalyan and Yu.A.Simonov, Yad.Fiz. 3, 1032 (1966).

[5] A.M.Badalyan, F.Calogero and Yu.A.Simonov, Nuovo Cimento. 68A, 572 (1970).

[6] A.I.Baz, Yu.T.Grin, V.F.Demin and M.V.Zhukov, Phys. of Element. Particles and Atom. Nucl., Vol.3, 275 (1972).

[7] Yu.F.Smirnov and K.V.Shitikova, Phys. of Element. Particles and Atom. Nucl., Vol.8, 847 (1977).

[8] V.I.Kukulin and V.M.Krasnopol'sky, J.Phys. G3, 795 (1977).

[9] K.Varga and Y.Suzuki, Phys.Rev. C52, 2885 (1995).

[10] J.Wurzer and H.M.Hofmann, Phys.Rev. C55, 688, (1997).

[11] B.S.Pudliner, V.R.Pandharipande, J.Carlson, S.C.Pieper and R.B.Wiringa, Phys.Rev. C56, 1720 (1997).

[12] R.A.Eramzhyan, G.G.Ryzhikh and Yu.M.Tchuvil'sky, Yad.FIz. 62, 42 (1999).

[13] A.I.Steshenko, Phys.of Atom.Nucl. 60, 520 (1997).

[14] P.Ring and P.Schuck, The Nuclear Many-Body Problem (Springer-Verlag, 1980).

[15] M.Abramowitz and I.A.Stegun, Handbook of Mathematical Functions (National Burean of Standards, Applied mathematic series - 55, USA, 1964).

[16] P.O.Löwdin, Phys.Rev. 97, 1474 (1955).

[17] D.A.Varshalovich, A.N.Moskalev and V.K.Chersonskij, Kvantovaja teorija uglovogo momenta (Nauka, Leningrad, 1975), p.228 (in Russian).

[18] A.B.Volkov, Nucl.Phys. 74, 33 (1965).

[19] D.M.Brink and E.Boecker, Nucl.Phys. A91, 1 (1967).

[20] I.V.Simenog and A.I.Steshenko, Ukr.Fiz.Zh. (Russ. Ed.) 38, 381 (1993). 


\section{FIGURES}

FIG. 1. Mass density of ${ }^{8} \mathrm{Be}$ as a function of the variables $z$ and $r_{\perp}=\sqrt{x^{2}+y^{2}}$, and contours of constant mass density in the section of ${ }^{8} \mathrm{Be}$ by the $(x, z)$ plane in the intrinsic coordinate frame rigidly bound to the nucleus.

FIG. 2. Total energy of the ${ }^{8}$ Be nucleus as a function of the variational parameter $R$.

FIG. 3. $2 \alpha$-distance as a function of the parameter $R$. 


\section{TABLES}

TABLE I. The nucleus ${ }^{8}$ Be ground state in microscopic models, Volkov's NN-potential [16]. The models (N1-N8) are described in the text.

\begin{tabular}{|c|c|c|c|c|c|c|c|c|}
\hline Characteristics & $\mathrm{N} 1$ & $\mathrm{~N} 2$ & N 3 & $\mathrm{~N} 4$ & $\mathrm{~N} 5$ & $\mathrm{~N} 6$ & $\mathrm{~N} 7$ & N 8 \\
\hline$E, \mathrm{MeV}$ & -52.732 & -50.017 & -36.859 & -49.605 & -47.910 & -36.135 & -52.975 & -44.836 \\
\hline$U_{\text {Coul }}, \mathrm{MeV}$ & 3.145 & 3.339 & 3.422 & 3.353 & 3.366 & 3.446 & 3.154 & 3.279 \\
\hline$<r^{2}>^{1 / 2}, \mathrm{fm}$ & 2.52 & 2.33 & 2.19 & 2.31 & 2.30 & 2.15 & 2.52 & 2.38 \\
\hline$\tilde{a}_{1}=\tilde{b}_{1}$ & 1.27 & 1.22 & 1.49 & 1.29 & 1.32 & 1.60 & 1.22 & 1.27 \\
\hline$\Psi_{1}$ & 2.23 & 2.33 & 1.49 & 2.43 & 2.07 & 1.60 & 2.18 & 1.89 \\
\hline$\tilde{a}_{2}=\tilde{b}_{2}$ & 1.35 & 1.29 & 1.49 & 1.35 & 1.32 & 1.60 & 1.29 & 1.34 \\
\hline$\tilde{c}_{2}$ & 1.91 & 1.80 & 1.49 & 1.93 & 2.07 & 1.60 & 1.79 & 1.62 \\
\hline$a_{1}=b_{1}$ & 1.49 & & & & & & 1.47 & 1.57 \\
\hline$c_{1}$ & 1.59 & & & & & & 1.56 & 1.56 \\
\hline$a_{2}=b_{2}$ & 1.28 & & & & & & 1.25 & 1.30 \\
\hline$\Psi_{3}$ & 1.37 & & & & & & 1.36 & 1.36 \\
\hline$s$ & 1.28 & & & & & & 1.29 & 1.30 \\
\hline$R, \mathrm{fm}$ & 1.76 & & & & & & 1.73 & 1.53 \\
\hline$\tilde{\tilde{a}}_{1}=\tilde{\tilde{b}}_{1}$ & & 1.50 & 1.86 & & & & 1.50 & 1.51 \\
\hline$\Psi_{4}$ & & 2.70 & 1.86 & & & & 2.48 & 2.10 \\
\hline$\tilde{\tilde{a}}_{2}=\tilde{\tilde{b}}_{2}$ & & 1.53 & 1.86 & & & & 1.65 & 1.72 \\
\hline$\tilde{\tilde{c}}_{2}$ & & 2.28 & 1.86 & & & & 2.37 & 2.12 \\
\hline$w_{1}$ & 1.74 & 1.35 & 1.03 & 1.00 & 1.00 & 1.00 & 0.97 & 0.50 \\
\hline$w_{2}=w_{3}$ & 1.00 & & & & & & 0.69 & 0.21 \\
\hline$w_{4}$ & & 0.72 & 0.69 & & & & 0.42 & 0.34 \\
\hline
\end{tabular}


TABLE II. Calculated ${ }^{6} \mathrm{He}$ features. Energies $E, U_{\text {Coul }}$ are given in $\mathrm{MeV}$, rms radii, $\left\{a_{i}, b_{i}, c_{i}\right\}$, $R$ are given in $\mathrm{fm}$.

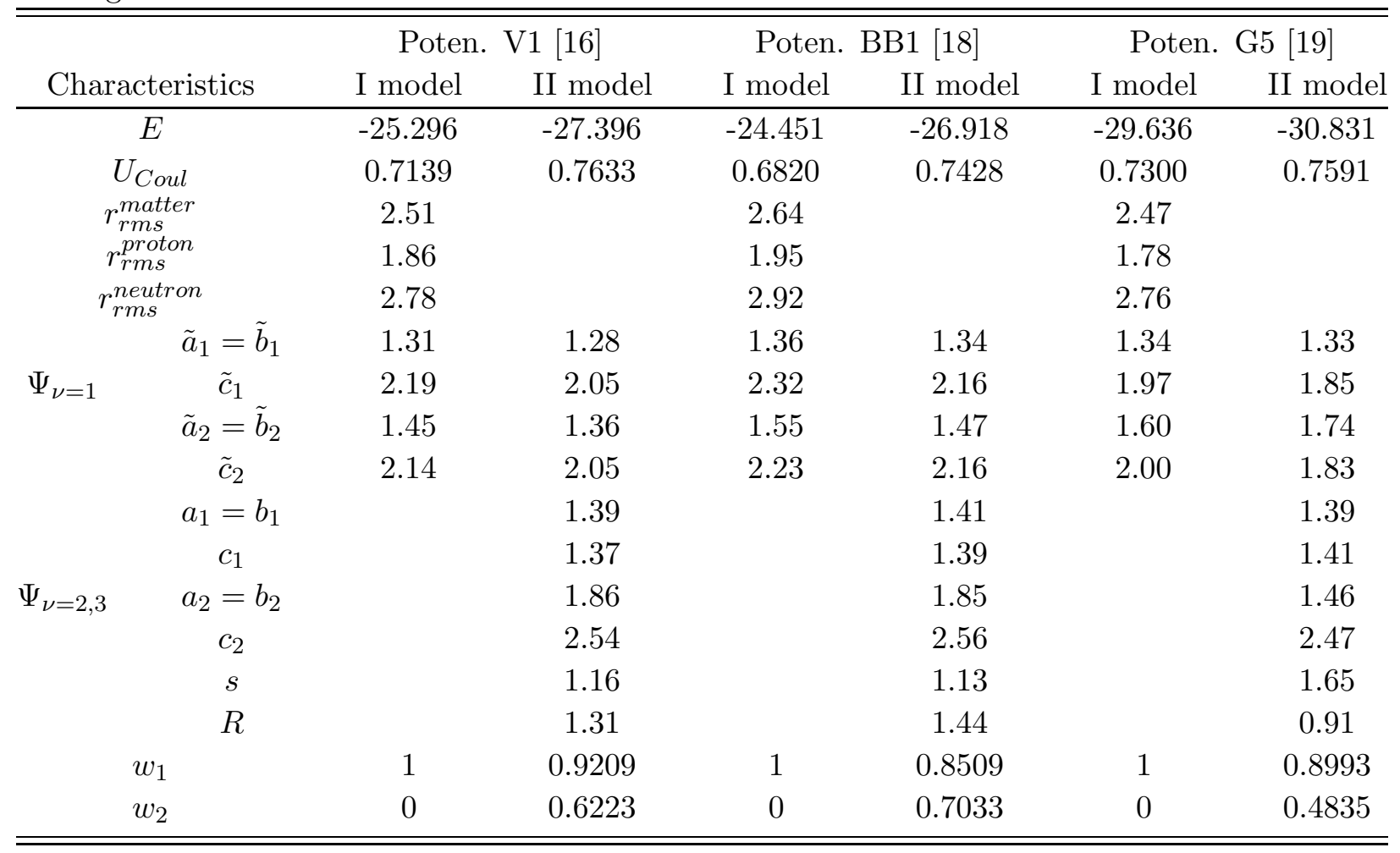




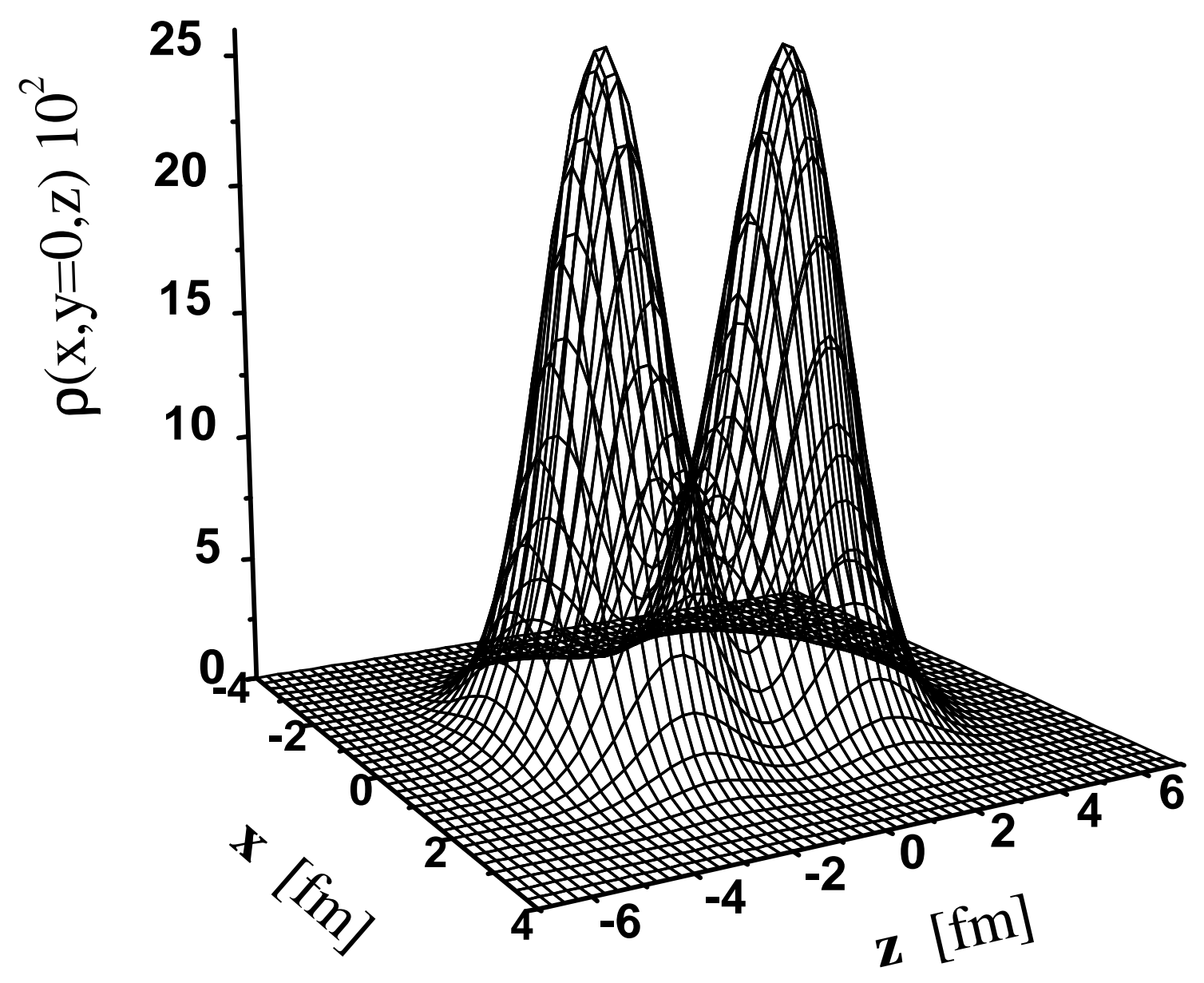




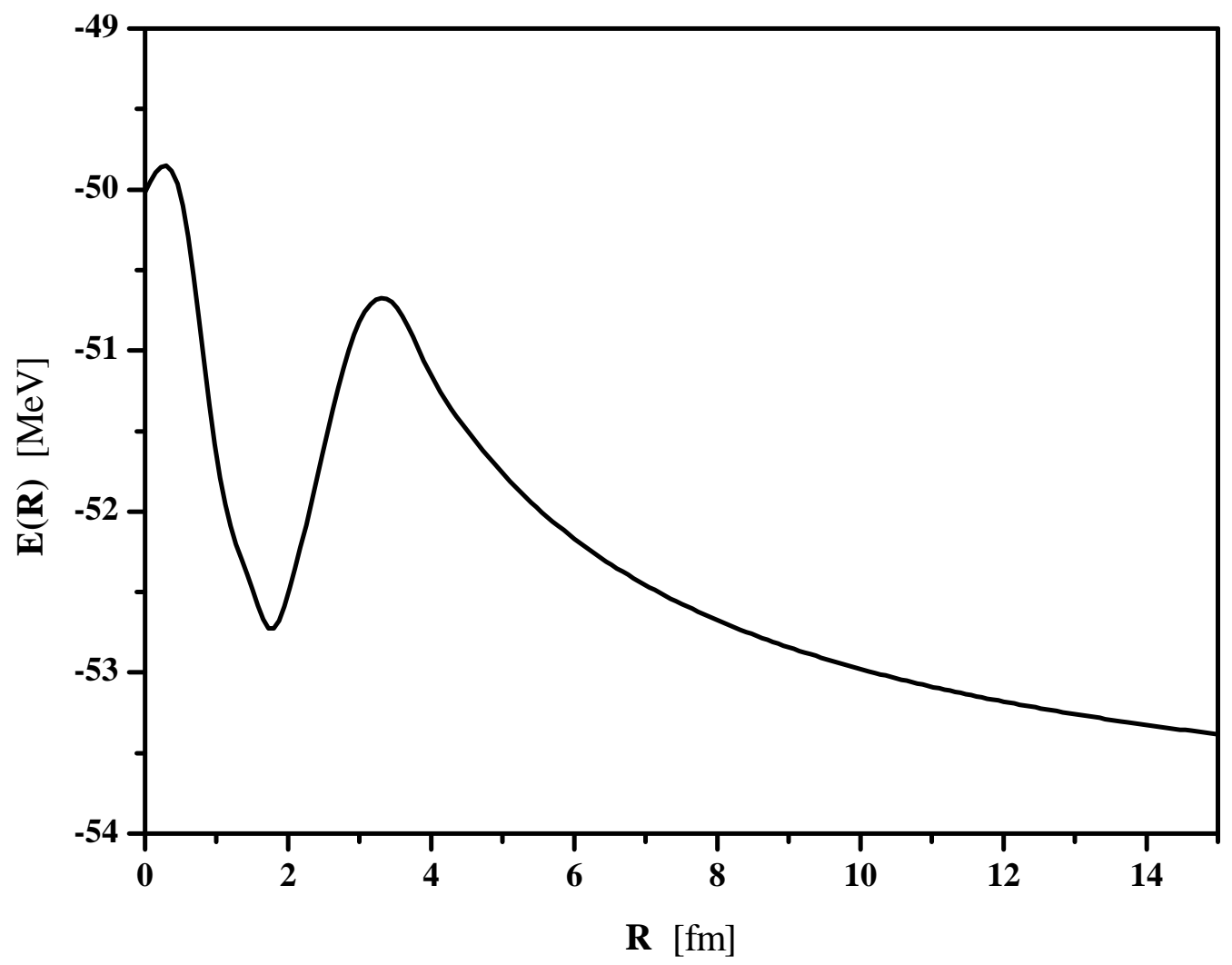




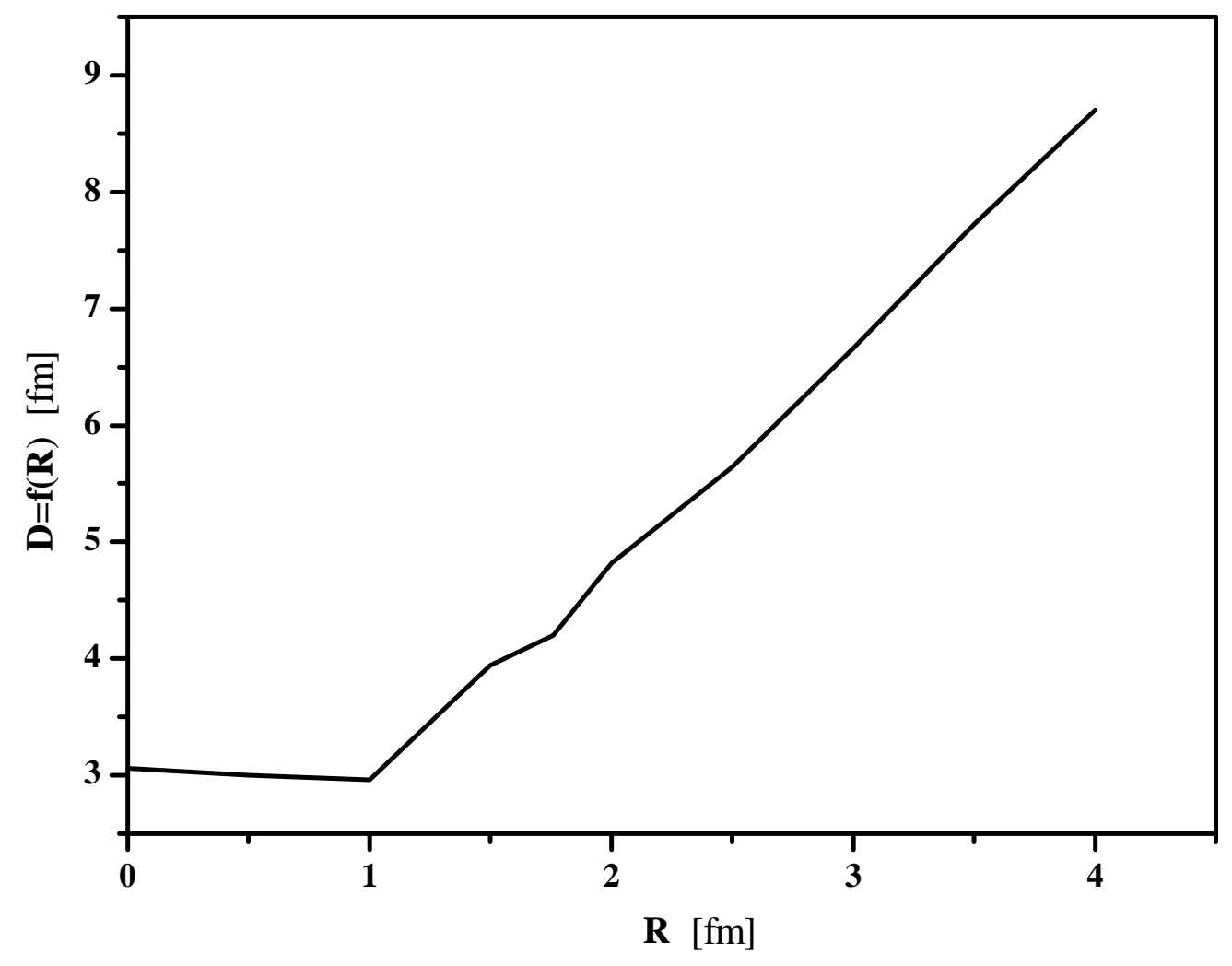

